\title{
Modal equity of accessibility to healthcare in Recife, Brazil
}

\author{
Boer Cui \\ McGill University \\ boer.cui@mail.mcgill.ca \\ Bernardo Serra \\ Institute for Transport Policy \& Develop- \\ ment-Brazil \\ bernardo.serra@itdp.org
}

\author{
Geneviève Boisjoly \\ Polytechnique Montréal \\ gboisjoly@polymtl.ca \\ Ahmed El-Geneidy \\ McGill University \\ ahmed.elgeneidy@mcgill.ca
}

\begin{abstract}
In the context of increasing urbanization and income inequality, transport professionals in the Global South need to be prepared to effectively plan for the needs of various groups within the population, particularly for those regarding health and well-being. Accessibility is widely used as a performance measure for land use and transport systems; it measures people's ease of reaching desired destinations and incorporates mode, time, and/or cost constraints. Considerable differences exist in the level of accessibility experienced by different mode users in reaching healthcare facilities, which calls for additional equity considerations given the prevailing socio-demographic characteristics of the users of various modes and the importance of healthcare facilities as a destination. In this study, we explore the distribution of accessibility to healthcare facilities by public transport and by car in Recife, Brazil, through an equity assessment to identify areas with low accessibility using these modes at different times of day. In general, the higher accessibility of public transport as well as greater modal equity was observed in central regions of Recife, whereas the periphery, where many low-income census tracts can be found, experiences significant inequity when it comes to access by both modes to healthcare facilities. This analysis allowed us to classify locations to access impoverished, access absolutely impoverished, and access impoverished by public transport areas, which can be targeted with appropriate land use and public transport policy interventions. This paper can be of value to professionals and researchers working toward equitable land use and transport systems in the Global South.
\end{abstract}

\section{Article history:}

Received: September 27, 2021

Accepted: November 10, 2021

Available online: January 24,

2022

\section{$1 \quad$ Introduction}

Universal health coverage and access are increasingly discussed by the World Health Organization (WHO) as key goals to improve population health (Evans et al., 2013). According to the WHO, universal access has three distinct dimensions: physical accessibility, financial affordability, and acceptability. Given the mounting availability of tools and methods to compute accessibility, the ease to reaching destinations, an increasing body of literature has emerged to quantify accessibility to healthcare from a land use and transport perspective, and its impact on health. While cities in the Global South are typi-

Copyright 2022 Boer Cui, Geneviève Boisjoly, Bernardo Serra \& Ahmed El-Geneidy

http://dx.doi.org/10.5198/jtlu.2022.2103

ISSN: 1938-7849 | Licensed under the Creative Commons Attribution - Noncommercial License 4.0

The Journal of Transport and Land Use is the official journal of the World Society for Transport and Land Use (WSTLUR) and is published and sponsored by the University of Minnesota Center for Transportation Studies. This paper is also published with additional sponsorship from WSTLUR. 
cally characterized by socio-spatial segregation and inequities, most studies on accessibility to healthcare have to date focused on the Global North.

The aim of this paper is to assess accessibility to healthcare in Recife, Brazil from a social and modal equity perspective. To do so, accessibility to healthcare facilities, by car and by public transport is calculated at four times of the day $(7 \mathrm{am}, 12 \mathrm{pm}, 5 \mathrm{pm}$ and $10 \mathrm{pm})$. The ratio between accessibility by public transport and accessibility by car is then calculated. The three measures (accessibility by car, by public transport, and the ratio) are then combined with income and density data to identify areas that should be prioritized in terms of land use and/or transport interventions. Not only do we examine the intermodal dimension of equity through the use of the ratio of accessibility, we also incorporate considerations of social equity to assess the income distribution of areas experiencing low accessibility and/or low ratio of accessibility to identify whether the low-income groups are more impacted. This study contributes to literature on transport equity in the Global South and is of relevance to researchers and planners wishing to adopt methods to support equity-based planning.

\section{$2 \quad$ Literature review}

\subsection{Transport inequities in Latin America}

In the last decade, researchers have shed light on the significant transport inequities that exist in several metropolitan regions in Latin America. Combined with the important socio-spatial segregation (Blanco et al., 2018; Keeling, 2008), the lack of adequate transport infrastructure results in important discrepancies in the provision of land use and transport benefits. Namely, a number of studies have demonstrated that low-income households tend to have lower accessibility to public transport services and to opportunities (Bocarejo \& Oviedo, 2012; Boisjoly et al., 2020; Delmelle \& Casas, 2012; Hernandez, 2018; Hernandez \& Rossel, 2015; Jaramillo et al., 2012). For example, in a study conducted in Montevideo, Uruguay, Hernandez (2018) demonstrates that individuals residing in low-income areas had significantly lower access to jobs and education opportunities. Similarly, Boisjoly et al. (2020) found that low-income households had lower accessibility to jobs, by public transport, and to rapid transit than high-income households in four metropolitan regions in Brazil. Furthermore, in a recent study conducted in Rio de Janeiro, Pereira (2018) found that the recent investments in public transport services have exacerbated the existing urban inequalities.

Recent research has demonstrated that the inequitable land use and transport patterns have significant impacts on low-income households. Several studies found that low-income individuals tend to have longer travel times and lower travel speeds to reach spatially distributed opportunities (Hernandez, 2018; Martínez et al.,2018; Vasconcellos, 2018). This is in part due to the fact that they mainly rely on public transport and active modes for their everyday mobility. In line with this, the lack of public transport has been shown to be associated with suppressed trips and activities (Falavigna \& Hernandez, 2016; Ureta, 2008; Vasconcellos, 2018). With respect to the Brazilian context, Vasconcellos (2018) found that low-income households have higher immobility rates due to lack of public transport options. Public transport provision is therefore inherent to discussions on transport equity in the Global South.

\subsection{Accessibility to healthcare}

Researchers working on accessibility to healthcare are typically concerned with two distinct interpretations of accessibility (Guagliardo, 2004; Wilson \& Rosenberg, 2004). The first one is the potential access to healthcare services, which refers to the geographic distribution and supply of healthcare services. The second one, the realized access, refers to actual utilization rates (Joseph \& Phillips, 1984). While distinct in nature, the literature has demonstrated a strong relationship between the potential access 
(referred to in this paper as accessibility to healthcare) and the health outcomes and service utilization. Researchers have demonstrated that the distance to healthcare facilities leads to a lower probability of using healthcare services, which contributes to increasing existing health inequities (Guagliardo et al., 2004; Haynes et al., 2003; Hiscock et al., 2008; Korda, et al., 2007).

More recently, researchers have worked on bridging the gap between health and transport geography studies to improve measurement and understanding of accessibility to healthcare. While several studies have been undertaken (Apparicio, et al., 2008; Law, et al., 2011; Mao \& Nekorchuk, 2013; Neutens, 2015; Paez, et al., 2010), they have typically focused on the Global North. Fewer studies have addressed this issue in the Global South context, namely because of the difficulty to obtain the required data. While previous research has highlighted existing health and transport inequities in Brazil (Macinko \& Lima-Costa, 2012), this study is, to our knowledge, the first to measure accessibility to healthcare based on travel times by car and by public transport in a Brazilian city. Investigating public transport accessibility is central to this study as the most recent travel survey of Recife reported that the majority of trips (55\%) for medical consultation were made by public transport or active modes (Grande Recife Consórcio de Transporte Metropolitano, 2019). The study proposes a method to investigate accessibility to healthcare by public transport and car using data that is available to researchers and planners. It presents a timely and novel empirical contribution that is of relevance to researchers, planners, and decision makers, while at the same time demonstrating the opportunities and limitations of the available data.

\section{Data and methodology}

\subsection{Area and unit of analysis}

The area of study is the municipality of Recife as shown in Figure 1, which consists of six political administrative regions (RPAs) which are further divided into 94 neighborhoods (bairros) and 1,843 census tracts. Due to computational, time and financial limitations, we needed to limit the number of points for the generation of accessibility measures. To do so, we overlaid a grid consisting of $1 \mathrm{~km} \mathrm{x} 1 \mathrm{~km}$ grid cells over the map of the municipality and then intersected with the municipality boundary, giving us 267 grid cells. The grid cells were then used as the unit of analysis for accessibility measures where the travel times between grid cells as well as the number of healthcare facilities present in each grid cell were calculated at the grid cell centroids. The resulting accessibility measures were interpolated using the inverse distance weighting method with the four closest neighbors to assign an accessibility value to each census tract at their centroids as other information such as density and average household income was only available at the census tract level.

\subsection{Data}

In order to generate accessibility measures to healthcare facilities, information on the location of healthcare facilities as well as travel times between grid cells were needed. The healthcare facilities considered in this analysis includes all hospitals as well as healthcare clinics located in the Recife municipality. The names and coordinates of healthcare facilities were obtained through the National Health Facility Register (Cadastro Nacional de Estabelecimentos de Saúde) (Ministério da Saúde, 2019) provided by the Ministry of Health (Ministério da Saúde) and then geocoded using Google Geocoding API (Google, n.d.). The locations of these healthcare facilities (1772 in totals) are shown in Figure 1 where we can observe an uneven distribution throughout the region. Specifically, there is a higher concentration of facilities in the central area of the municipality (namely the intersection of the first four RPAs) as well in the seaside communities located in the southern region. There are also noticeably very few facilities in the northern and western peripheries of Recife which may reflect the lower density in these areas. 
Travel times by public transport and by car were collected using the Google Maps Distance Matrix API (Google, n.d.). This Google API returns the public transport travel time, in minutes, of the fastest route requiring the lowest number of transfers ("fewer transfers" transit routing preference) between each origin-destination pair from an O/D matrix consisting of all 267 grid cells. The public transport travel times generated by the Google API include access, in-vehicle, transfer and egress times and excludes the waiting time prior to departure. Not accounting for waiting time prior to departure might underestimate accessibility variations throughout the day, potentially more in peripheral areas with lower public transport frequency outside peak hours or in areas characterized by high-frequency services specifically at peak hours. In doing so, we hypothesize that individuals are flexible in terms of their departure time. Generating travel times using GTFS data would have allowed for more customization to include waiting times but this information was not available for Recife. It is only until recently that GTFS data has become more readily available for metropolitan regions in the Global South, particularly in South America as few studies have made use of this data, for example that of Rio de Janeiro by Pereira, et al., (2019). Frequency fluctuations could certainly be investigated in further research in terms of accessibility by public transport and modal equity, namely to understand whether low-income areas are more penalized by low frequencies of public transport services at specific points of the day.

Car travel times generated using this API were based on the pessimistic traffic model, which is defined as a conservative model. This means that travel times estimated with this assumption consider traffic conditions that reflect an above average congestion level (Google Maps Platform, 2021). Note that given the proprietary nature of the tool, the exact details of the algorithm are not available. The pessimistic traffic model was selected to account for uncertainties in travel times. The travel times were obtained for 7 a.m. (representative of the morning peak period where public transport service may be most frequent and traffic congestion on the roads is worst), noon (less frequent service and less congestion), 5 p.m. (frequent service and increased congestion) and 10 p.m. (infrequent service and no congestion). Generating multiple accessibility measures at various times provides a more comprehensive picture of how accessibility as well as modal equity fluctuates throughout the day depending on the availability and level of service of transport systems.

Additional data on the population density as well as average annual household income aggregated at the census tract level was obtained from the 2010 census (Instituto Brasileiro de Geografia e Estatistica, 2011). 


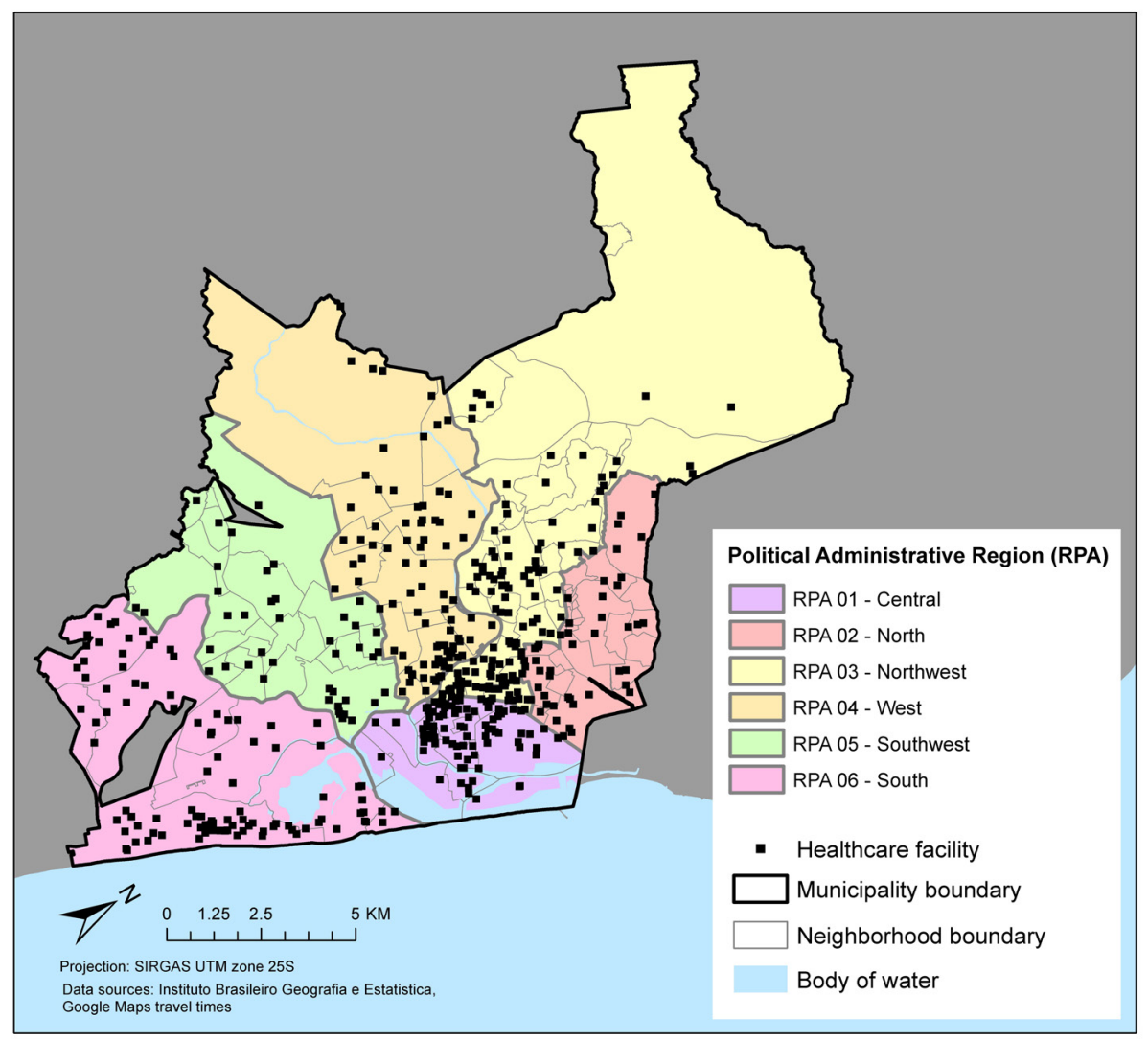

Figure 1. Location of healthcare facilities in the Recife municipality

\subsection{Accessibility}

\subsubsection{Accessibility to healthcare facilities}

Cumulative accessibility measures, measuring the number of healthcare facilities that can be reached from each grid cell by public transport and by car under a specified travel time threshold, were generated in this study. Cumulative accessibility measures have been found to accurately represent the relative accessibility experienced across a metropolitan area and is adequate for planning purpose due to its ease of computation and interpretation compared to gravity-based accessibility measures. However, researchers studying accessibility to healthcare have recommended that accessibility measures using the two-step floating catchment area (2SFCA) method be used which controls for the travel impedance of access as well as capacity restrictions and competition effects of access to healthcare (Luo \& Wang, 2003). Unfortunately, the power of this method lies in knowing the capacity of healthcare facilities, which we do not have information about in this study, in order to generate a meaningful service-to-population ratio to account for potential demand for the service at a facility. Furthermore, as stated by Neutens (2015) in a review of the literature on equity and healthcare accessibility, simpler measures (that do not account for competition) are more often used in countries of the Global South since the spatial separation is typically a greater constraint than the competition when concerned with equity questions. For example, in an equity assessment of the new bus rapid transit system in Cali, Colombia, Delmelle and Casas (2012) measures the accessibility to healthcare without considering the competition effect. Therefore, in the current study, the cumulative measure is used instead which also makes interpreting the results of an assessment of modal equity easier. 
The cumulative measure is calculated as follows:

$$
\begin{aligned}
& A_{i, m}=\sum_{j=1}^{J} O_{j} f\left(C_{i j}\right) \\
& f\left(C_{i j}\right)=\left\{\begin{array}{l}
1 \text { if } C_{i j, m} \leq t_{\text {threshold }} \\
0 \text { if } C_{i j, m}>t_{\text {threshold }}
\end{array}\right.
\end{aligned}
$$

where $A_{i, m}$ is the accessibility at grid cell $i$ to healthcare facilities by mode $m, O_{j}$ is the number of healthcare facilities in grid cell $j, f\left(C_{i j}\right)$ is the weighting function where $C_{i j, m}$ is the time cost of travel from the centroid of $\mathrm{i}$ to centroid of $j$ using mode $\mathrm{m}$ and $t_{\text {threshold }}$ is the travel time threshold.

As mentioned above, accessibility to healthcare facilities was calculated at the centroids of $1 \mathrm{~km} \mathrm{x} 1$ $\mathrm{km}$ grid cells and then interpolated at the census tract centroids. The healthcare facilities are considered accessible from a grid cell if they can be reached within the travel time threshold between that grid cell centroid and the centroid of the grid cell in which the facilities are located in. In this study, a travel time threshold of 45 minutes was used to generate accessibility by public transport and by car to compare modal equity. The 45-minute threshold was selected to reflect local travel times, but is also frequently used as a reference in accessibility studies (Boisjoly \& El-Geneidy, 2017). While this threshold underestimates the public transport travel time and overestimates the car travel time for many commuters in Recife based on the results found in the Recife Origin/Destination Survey (Grande Recife Consórcio de Transporte Metropolitano, 2019), this serves as an adequate compromise between the two modes. In addition, 45 minutes was also used as the travel time threshold in previous studies of modal equity (Golub \& Martens, 2014).

\subsubsection{Ratio of accessibility to healthcare facilities}

The ratio of accessibility was introduced by Golub and Martens (2014) and is measured as the ratio of public transport accessibility to healthcare facilities to car accessibility to healthcare facilities:

$$
R_{i}=A_{i, P T} / A_{i, C A R}
$$

A ratio of accessibility less than one for a particular census tract implies that public transport accessibility is worse than car accessibility and vice-versa for a ratio of greater than one. A ratio close to one would imply modal equity in terms of accessibility.

\subsection{Identifying target areas for interventions}

While previous research on modal equity (Golub \& Martens, 2014) defined an access poverty line to be at a ratio of accessibility of 0.33 , we chose to use a tertile ranking to identify areas that are impoverished in terms of both absolute access by public transport and by car as well as modal equality. The use of a tertile ranking compares the characteristic of a particular census tract with all others in the study area for a specific time of day (i.e., 7 a.m., noon, 5 p.m. or 10 p.m.) for the measure that we are interested in (i.e., accessibility by public transport, accessibility by car, or ratio of accessibility). Values of accessibility or a ratio of accessibility in the first tertile are deemed low as compared to the second and third tertiles. Areas of low accessibility by both modes are those with a combined tertile ranking (i.e., multiplication of the tertile rankings of accessibility by public transport and by car) of one. We also consider density 
in this process by focusing on areas that are dense (where more people will be impacted by comparable interventions to improve accessibility) by excluding areas with density in the lowest tertile. In other words, similar land use and transport interventions (e.g., new bus service, new healthcare service) can benefit a greater number of individuals in a denser area, given their proximity. This allows concentrating on urban or peri-urban areas (as can be observed in the result section), where public transport services play a key role in ensuring access to destinations. Other studies could focus on rural contexts in an attempt to identify potential strategies to mitigate the effect of low accessibility to healthcare, namely by public transport.

It is important to mention that an arbitrary decision had to be made regarding the accessibility and ratio thresholds, since there exists no theoretical or empirical justification as to what is considered acceptable or adequate. From a sufficientarism perspective, the thresholds would reflect the minimum level required for individuals to meet their needs (Lucas et al., 2016), which many argue depends on the context (Pereira et al., 2017). In the absence of guidelines on this minimum level, a ranking approach (in this case tertile) was selected to reflect the local context. This is also in line with the theoretical perspective stating that the minimum level should be maximized (Pereira et al., 2017). As such, the ranking approach allows identifying areas experiencing the lowest accessibility or ratio. The specific threshold (here the first tertile) could be selected depending on the objectives of a region. Also, future studies could be conducted to evaluate the outcomes (e.g., healthcare consultations, health outcomes, etc.) associated with different levels and ratios. Finally, a context-specific assessment of the accessibility and ratio distributions, together with a sensitivity analysis, could be conducted to provide additional guidance on which threshold to select.

\section{$4 \quad$ Analysis and discussion}

\subsection{Access to healthcare facilities by public transport and car}

A comparison of accessibility to healthcare facilities by public transport and by car at various times throughout the day is shown in Figure 2. The maximum number of healthcare facilities that can be accessed by a census tract is 1,164 , which is only possible by car. Accessibility by public transport is highest in the central region of Recife and decreases radially outwards at all times during the day. It also does not appear to be closely correlated with the location of rapid transit stations (except slightly in the southern region). This may be attributed to the density of healthcare facilities in the central regions which allows individuals, using less efficient modes of public transport to access them whereas there are fewer hospitals to be accessed in the regions where rapid transit operates. We expected accessibility to change throughout the day and especially a significant decrease at 10 p.m. due to a reduction in service. However, this was not observed for almost the entire region except for a few areas in the north and northwestern regions. On the other hand, accessibility by car changes throughout the day. It is higher in the central regions of Recife and decreases radially westwards during the morning and afternoon peaks which could be attributed to the higher density of healthcare facilities located in this area despite more traffic congestion. Accessibility by car becomes more evenly distributed at noon and at 10 p.m., where accessibility by car is uniformly very high in almost all regions which could be attributed to less traffic congestion.

Figure 3 presents census tracts with low accessibility to healthcare facilities by public transport and by car differentiated by average annual household income at various times throughout the day. It is clear that, without accounting for density (3a), areas on the periphery of the municipality experience low accessibility by both modes due to a lack of healthcare facilities and underdeveloped transport systems. 
Interestingly, a few census tracts located in the southern corner of the municipality experience low accessibility by both modes at all times during the day except for 7 a.m., which could be that this area is not strongly affected by traffic congestion compared to other parts of Recife during the morning peak. Despite experiencing low accessibility in general, it appears that in comparison with other places, the accessibility of these census tracts is actually quite good at $7 \mathrm{a} . \mathrm{m}$. After excluding the sparsely populated areas (3b), the remaining census tracts are access impoverished and should be prioritized for land-use interventions to increase the number of healthcare facilities available in these areas which would improve their accessibility to be comparable to the rest of Recife. We observe that many of these census tracts have average annual household incomes of less than $\mathrm{R} \$ 15,000$ which is approximately half of the annual household income average across census tracts in the Recife municipality. This represents an important consideration of social equity to ensure that more vulnerable groups, who depend more heavily on public transport, have comparable access to healthcare compared to the rest of the population.
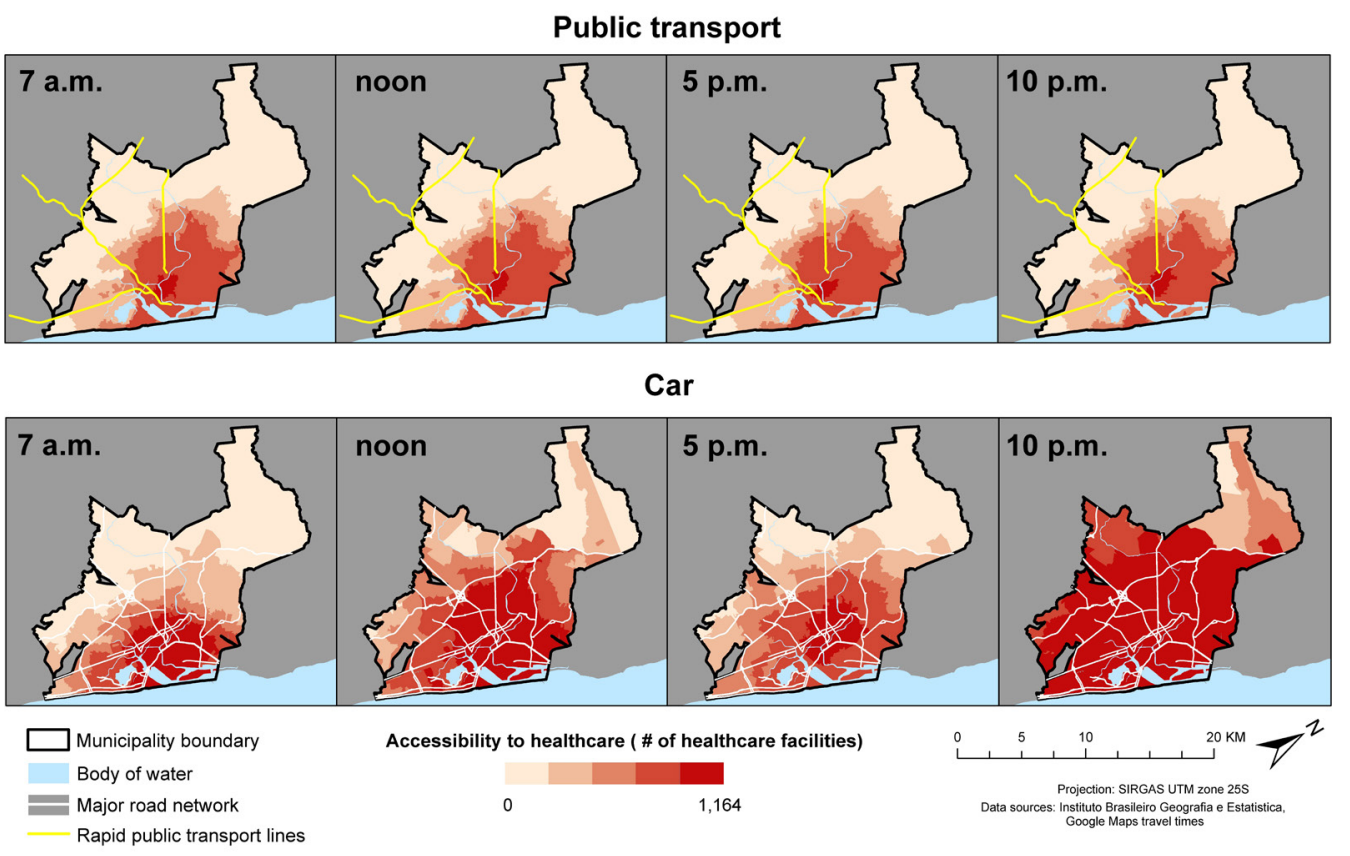

Figure 2. Accessibility to healthcare by public transport and car at various times throughout the day 


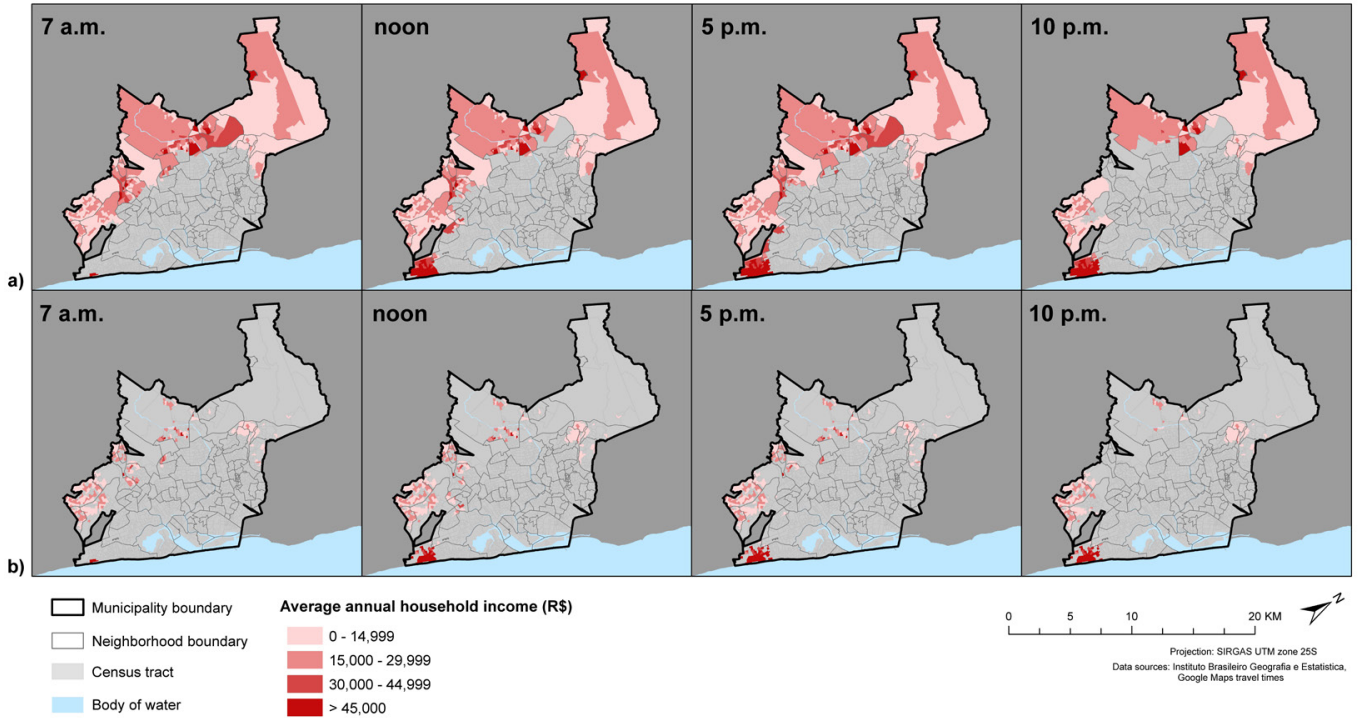

Figure 3. Average annual household income of census tracts, a) regardless of density, b) considers only dense areas, with low accessibility by public transport and car at various times throughout the day

\subsection{Ratio of accessibility to healthcare}

The ratio of accessibility to healthcare facilities in each census tract depending on the time of day is presented in Figure 4. The biggest difference is observed for the 7 a.m. period or the morning peak where the ratio of accessibility is much higher than one in more central regions of the north, northwestern and western RPAs compared to other areas of the municipality. These are areas where accessibility to healthcare facilities by public transport is much better than by car, likely due to the effects of morning congestion. This is in accordance with Figure 2 where we see that the region with a very high ratio of accessibility in Figure 4 coincides with the area in Figure 2 where car accessibility is much lower than public transport. Accessibility ratios are then fairly consistent from noon to $10 \mathrm{p} . \mathrm{m}$. and we see that there are no areas where public transport accessibility is much higher than car accessibility. As well, relative modal equity is achieved in more central areas of Recife and becomes more inequitable further from the center. The trend is particularly pronounced at 10 p.m.

Figure 5 presents census tracts with ratios of accessibility in the lowest tertile at different times throughout the day where the top row includes areas of all densities (5a) and the bottom excludes low densities (5b). The colors represent the average annual household income of the census tract following the same categories as Figure 3. We observe that many census tracts in Figure 5 overlap with those shown in Figure 3, where both accessibility by public transport and by car are low yet public transport is even lower, making these areas completely impoverished in terms of accessibility (access absolutely impoverished areas). For these areas, land-use interventions would be more impactful to bring healthcare facilities closer to individuals rather than to try to achieve modal equity.

Further, areas that do experience high levels of modal inequity with ratios of accessibility in the lowest tertile but with medium or high combined accessibility by car and by transit are shown are presented 
in Figure 6 (considering only dense census tracts). This mainly reflects areas that have a low accessibility by public transport, but a relatively high level of accessibility by car. These areas (access impoverished by public transport) are areas where investments in public transport would help minimize modal inequity in the region. At the same time, we should also focus on areas where a greater proportion of residents may depend on public transport which tend to be correlated with areas that have very low average household income $(<\mathrm{R} \$ 15,000)$.

There are some temporal differences for where modal inequity is observed throughout the day, which means that improvements with regards to the existing public transport service should be adjusted for different regions at different times of the day. One important note to add is that while we observe at 7 a.m. that there are some higher-income census tracts in southern Recife that are considered access impoverished by public transport and where improvements to the public transport system are called for, we do not know whether wealthier residents in these areas actually use and rely on public transport. Meanwhile, from noon to 10 p.m., census tracts, many of which have below-average annual household income and therefore are places where more residents depend on public transport, experience modal inequity. As such, an analysis of the demand for public transport in the access impoverished census tracts shown in Figure 6 should be done to maximize the benefits of public transport investments, in terms of improved service or expanded system coverage, for users as well as the transportation agency.
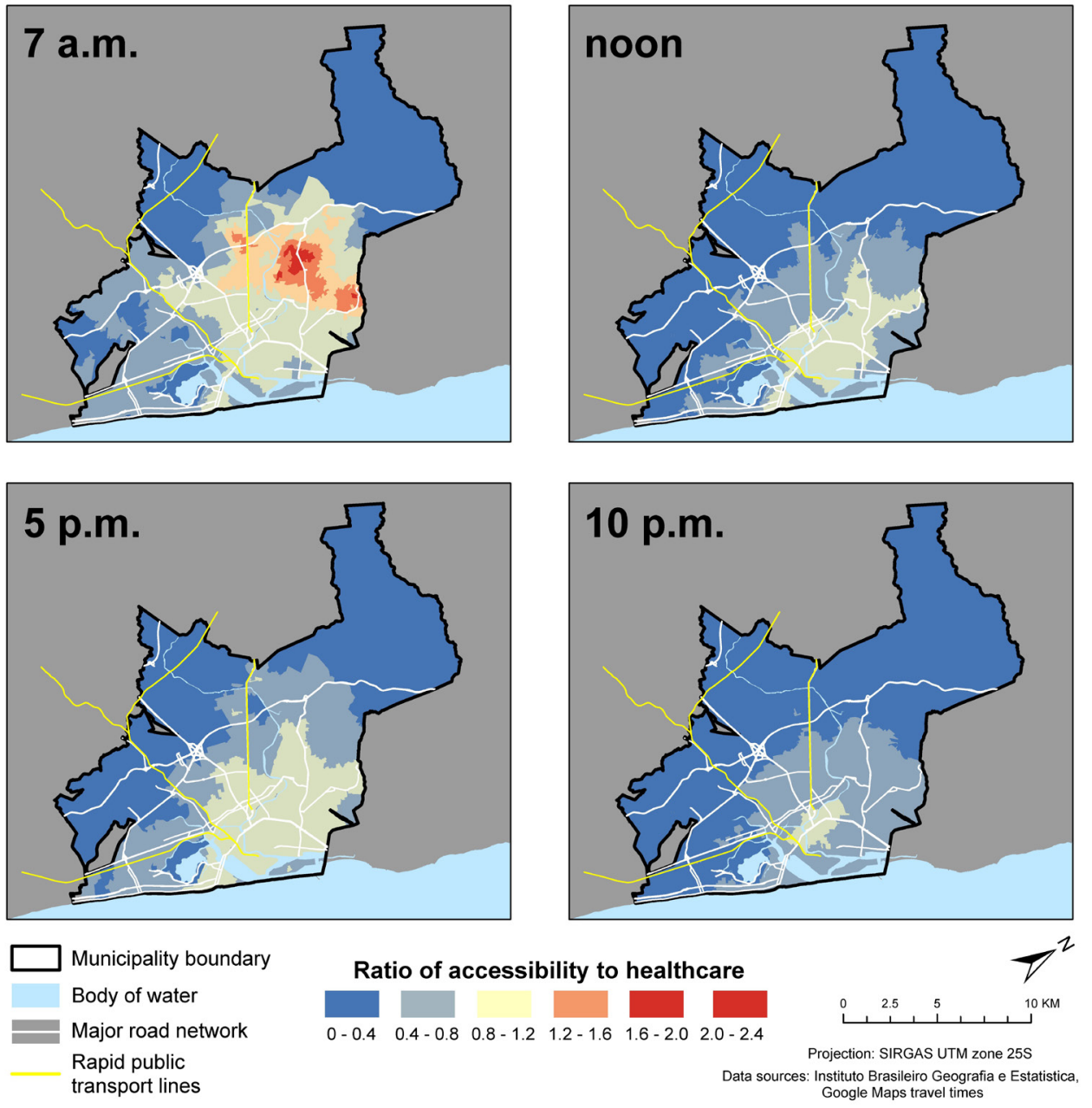

Figure 4. Ratio of accessibility to healthcare at various times throughout the day 


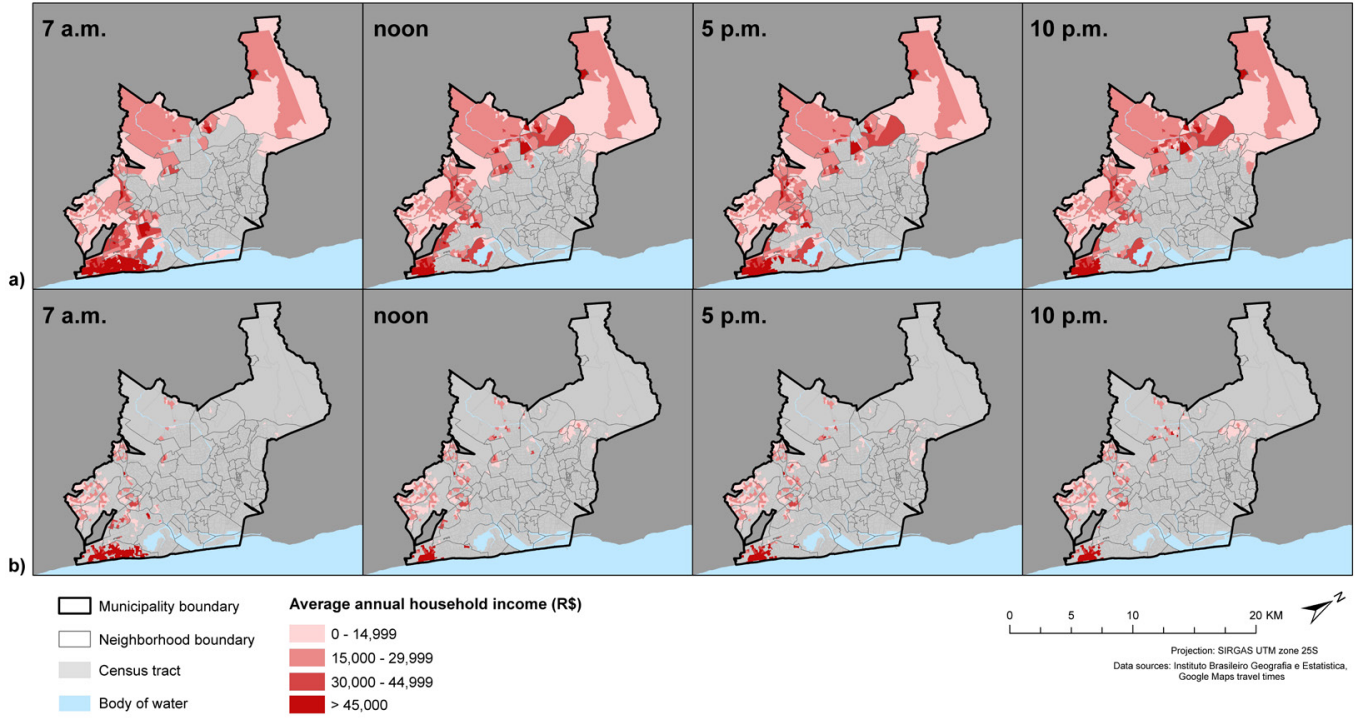

Figure 5. Access absolutely impoverished areas - Average annual household income of census tracts with low accessibility ratio at various times throughout the day, a) regardless of density, b) considering only dense areas
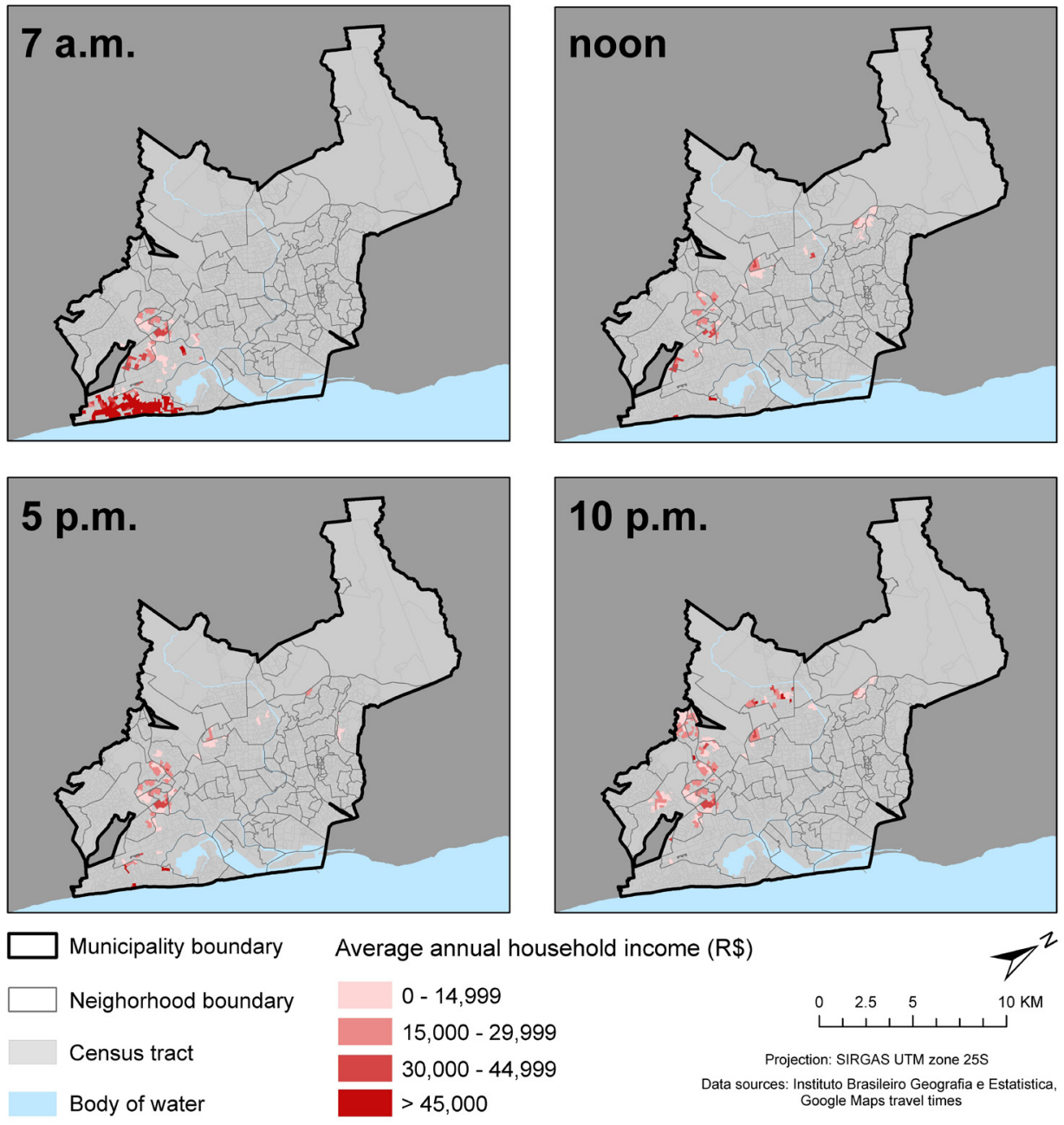

Figure 6. Access impoverished by public transport - Average household income of dense census tracts with very low accessibility ratio but medium or high combined accessibility by transit and car, at various times throughout the day 


\section{Conclusion}

As the Global South is currently experiencing high rates of urbanization as well as high levels of income inequalities, land use and transport systems play an important role in mediating existing inequities in order to improve quality of life for all, of which access to healthcare plays an important role. Access to healthcare is dependent on the mode used, which prompts further equity considerations as those who depend on public transport more heavily are typically those in more vulnerable groups. In this study, we explored the distribution of accessibility to healthcare facilities for census tracts in the municipality of Recife, Brazil, while considering modal equity through the use of a ratio of accessibility to measure the gap between accessibility by public transport and by car. Social equity was also considered as we presented the average annual household income of census tracts to identify lower-income areas which should be further targeted for interventions. Temporal differences were also explored using accessibility measures generated at different times of the day.

There are three categories of access impoverishment which we have identified in this study. The first is access impoverished areas which include census tracts that are sufficiently dense with low accessibility to healthcare facilities by public transport and by car. Access absolutely impoverished areas include dense census tracts with low accessibility to healthcare by both modes as well as having low ratios of accessibility. Areas that are access impoverished by public transport are dense census tracts that have low ratios of accessibility but not low accessibility by both modes. Consequently, the appropriate intervention for each group is different where for access impoverished and access absolutely impoverished census tracts, placing more facilities within these areas would be more impactful to increase the general accessibility. On the other hand, improvements to the public transport service or expansion of the network is preferred in access impoverished by public transport areas to minimize the gap between accessibility by public transport and by car. In all these cases, areas of lower average household income should be prioritized further for a more equitable distribution of transport services.

A major limitation associated with this study is the unavailability of information on the capacity or number of services offered at each healthcare facility. The use of the cumulative accessibility measure which counts the number of healthcare facilities that are accessible would lead one to believe that the more facilities that can be accessed, the better. However, this does not account for demand for healthcare services at these facilities, like in the calculation of a service-to-demand ratio using the 2SFCA method. For example, absolute accessibility by public transport and by car is higher in the central region as there are more healthcare facilities concentrated in these areas, but it is expected that population density is also the highest which has a negative impact on accessibility. On the other hand, the use of this data would make interpretation of the results of modal equity more complex as it requires the differentiation of competition by mode (i.e., commuters that would access hospitals using public transport compared to those that would use car). Future studies on accessibility to healthcare in the Global South should make use of more detailed data on the capacity of healthcare facilities at the same time as information from Origin/Destination surveys which would allow researchers to model realistically the mode used by commuters to access healthcare. Further, all clinics and hospitals are aggregated in the same mea-

sure, although there exists a wide variety of services offered in the different types of facilities (from local health clinics to specialized hospitals). This could also be investigated in further research, namely by disaggregating the analysis by types of facilities, thereby allowing a more detailed understanding of the accessibility to healthcare services in the region. Nonetheless, the method proposed in this paper allows analyzing the physical barriers to accessing healthcare. It is important to mention that other dimensions of access are also crucial to the utilization of healthcare such as affordability and acceptability which can be incorporated in a more comprehensive accessibility measure. 
Another limitation lies in the use of the Google Distance Matrix API to generate public transport travel times rather than GTFS data as it is unclear how Google calculates travel times in the absence of openly available GTFS data. Having more data about actual travel schedules and reliability to provide a more realistic picture of accessibility may help to explain why accessibility by public transport does not fluctuate much throughout the day, especially at 10 p.m. It would also be helpful in better accounting for waiting times, namely by considering headway times. Nonetheless, this study proposes a method to include equity concerns when aiming to increase accessibility to healthcare.

\section{Acknowledgements}

This research was partially funded by the Natural Sciences and Engineering Research Council of Canada (NSERC) and the Social Sciences and Humanities Research Council of Canada (SSHRC). The authors would like to thank Guillaume Barreau for his help in generating the travel times by public transport and by car. 


\section{References}

Apparicio, P., Abdelmajid, M., Riva, M., \& Shearmur, R. (2008). Comparing alternative approaches to measuring the geographical accessibility of urban health services: Distance types and aggregationerror issues. International Journal of Health Geographics, 7(7).

Blanco, J., Lucas, K., Schafran, A., Verlinghieri, E., \& Apaolaza, R. (2018). Contested mobilities in the Latin American context. Journal of Transport Geography, 67, 73-75.

Bocarejo, J., \& Oviedo, D. (2012). Transport accessibility and social inequities: A tool for identification of mobility needs and evaluation of transport investments. Journal of Transport Geography, 24, 142-154.

Boisjoly, G., \& El-Geneidy, A. (2017). Measuring performance: Accessibility metrics in metropolitan regions around the world. Washington, DC: Brookings Institution. Retrieved from https://www.brookings. edu/wp-content/uploads/2017/08/measuring-performance-accessibility-metrics.pdf

Boisjoly, G., Serra, B., Oliveira, G., \& El-Geneidy, A. (2020). Accessibility measurements in São Paulo, Rio de Janeiro, Curitiba and Recife, Brazil. Journal of Transport Geography, 82, 102551.

Delmelle, E., \& Casas, I. (2012). Evaluating the spatial equity of bus rapid transit-based accessibility patterns in a developing country: The case of Cali, Colombia. Transport Policy, 20, 36-46.

Evans, D., Hsu, J., \& Boerma, T. (2013). Universal health coverage and universal access (Bulletin of the World Health Organization). Paris :OECD

Falavigna, C., \& Hernandez, D. (2016). Assessing inequalities on public transport affordability in two latin American cities: Montevideo (Uruguay) and Córdoba (Argentina). Transport Policy, 45, 145155.

Golub, A., \& Martens, K. (2014). Using principles of justice to assess the modal equity of regional transportation plans. Journal of Transport Geography, 41, 10-20.

Google. (n.d.). Distance matrix API. Retrieved from https://developers.google.com/maps/documentation/distance-matrix/intro

Google. (n.d.). Geocoding API. Retrieved from https://developers.google.com/maps/documentation/ geocoding/intro

Google Maps Platform. (2021). Distance Matrix API. Retrieved from https://developers.google.com/ maps/documentation/distance-matrix/overview\#traffic-model

Grande Recife Consórcio de Transporte Metropolitano. (2019). Pesquisa origem-destino metropolitana 2018. Retrieved from http://planodemobilidade.recife.pe.gov.br/node/61265

Guagliardo, M. F. (2004). Spatial accessibility of primary care: Concepts, methods and challenges. International Journal of Health Geographics, 3(3).

Guagliardo, M. F., Ronzio, C. R., Cheung, I., Chacko, E., \& Joseph, J. G. (2004). Physician accessibility: An urban case study of pediatric providers. Health \& Place, 10(3), 273- 283.

Haynes, R., Lovett, A., \& Sunnenberg, G. (2003). Potential accessibility, travel time, and consumer choice: Geographical variations in general medical practice registration in Eastern England. Environment and Planning A, 35, 1733-1750.

Hernandez, D. (2018). Uneven mobilities, uneven opportunities: Social distribution of public transport accessibility to jobs and education in Montevideo. Journal of Transport Geography, 67, 119-125.

Hernandez, D., \& Rossel, C. (2015). Inequality and access to social services in Latin America: Spacetime constraints of child health checkups and prenatal care in Montevideo. Journal of Transport Geography, 44, 24-32.

Hiscock, R., Pearce, J., Blakely, T., \& Witten, K. (2008). Is neighborhood access to healthcare provision associated with individual-level utilization and satisfaction? Health Services Research, 43, 2183-2200. 
Instituto Brasileiro de Geografia e Estatistica. (2011). Demographic census 2010. Rio de Janeiro: Brazilian Institute of Geography and Statistics.

Jaramillo, C., Lizarraga, C., \& Luis Grindlay, A. (2012). Spatial disparity in transport social needs and public transport provision in Santiago de Cali (Colombia). Journal of Transport Geography, 24, 340-357.

Joseph, A. E., \& Phillips, D. R. (1984). Accessibility and utilization: Geographical perspectives on healthcare delivery. New York: Harper and Row Publishers.

Keeling, D. (2008). Latin America's transportation conundrum. Journal of Latin American Geography, $7(2), 133-154$.

Korda, R. J., Butler, J. R., Clements, M. S., \& Kunitz, S. J. (2007). Differential impacts of healthcare in Australia: Trend analysis of socioeconomic inequalities in avoidable mortality. International Journal of Epidemiology, 36, 157-165.

Law, M., Dijkstra, A., Douillard, J., \& Morgan, S. (2011). Geographic accessibility of community pharmacies in Ontario. Healthcare Policy, 6(3), 36-46.

Lucas, K., van Wee, B., \& Maat, K. (2016). A method to evaluate equitable accessibility: Combining ethical theories and accessibility-based approaches. Transportation, 43(3), 473-490.

Luo, W., \& Wang, F. (2003). Measures of spatial accessibility to healthcare in a GIS environment: Synthesis and a case study in the Chicago region. Environment and Planning B: Planning and Design, $30,865-884$.

Macinko, J., \& Lima-Costa, M. (2012). Horizontal equity in healthcare utilization in Brazil, 19982008. International Journal for Equity in Health, 11(1), 33.

Mao, L., \& Nekorchuk, D. (2013). Measuring spatial accessibility to healthcare for populations with multiple transportation modes. Health \& Place, 24, 115-122.

Martínez, C., Hodgson, F., Mullen, C., \& Timms, P. (2018). Creating inequality in accessibility: The relationships between public transport and social housing policy in deprived areas of Santiago de Chile. Journal of Transport Geography, 67, 102-109.

Ministério da Saúde. (2019). Cadastro nacional de estabelecimentos de Saúde. Rio de Janiero: Minsitry of Health of Brazil. Retrieved from: http://dados.gov.br/dataset/cnes

Neutens, T. (2015). Accessibility, equity and healthcare: Review and research directions for transport geographers. Journal of Transport Geography, 43, 14-27.

Paez, A., Mercado, R., Farber, S., Morency, C., \& Roorda, M. (2010). Accessibility to healthcare facilities in Montreal Island: An application of relative accessibility indicators from the perspective of senior and non-senior residents. International Journal of Health Geographics, 9(52), 1-15.

Pereira, R. (2018). Transport legacy of mega-events and the redistribution of accessibility to urban destinations. Cities, 81, 45-60.

Pereira, R., Banister, D., Schwanen, T., \& Wessel, N. (2019). Distributional effects of transport policies on inequalities in access to opportunities. Journal of Transport and Land Use, 12(1), 741-764.

Pereira, R., Schwanen, T., \& Banister, D. (2017). Distributive justice and equity in transportation. Transport Reviews, 37(2), 170-191.

Ureta, S. (2008). Mobilizing poverty? Mobile phone use and everyday spatial mobility among lowincome families in Santiago, Chile. The Information Society, 24(2), 83-92.

Vasconcellos, E. (2018). Urban transport policies in Brazil: The creation of a discriminatory mobility system. Journal of Transport Geography, 67, 85-91.

Wilson, K., \& Rosenberg, M. W. (2004). Accessibility and the Canadian healthcare system: Squaring perceptions and realities. Health Policy, 67, 137-148. 